\title{
Suppression of NLRX1 in chronic obstructive pulmonary disease
}

\author{
Min-Jong Kang, ${ }^{1,2}$ Chang Min Yoon, ${ }^{1}$ Bo Hye Kim, ${ }^{1}$ Chang-Min Lee, ${ }^{2}$ Yang Zhou, ${ }^{2}$ Maor Sauler, ${ }^{1}$ Rober Homer, ${ }^{1,3}$ Anish Dhamija, ${ }^{4}$ \\ Daniel Boffa, ${ }^{4}$ Andrew Phillip West, ${ }^{3}$ Gerald S. Shadel, ${ }^{3}$ Jenny P. Ting, ${ }^{5}$ John R. Tedrow, ${ }^{6}$ Naftali Kaminski, ${ }^{1}$ Woo Jin Kim, \\ Chun Geun Lee, ${ }^{2}$ Yeon-Mok Oh, ${ }^{8}$ and Jack A. Elias ${ }^{2}$ \\ 1Pulmonary, Critical Care and Sleep Medicine, Yale School of Medicine, New Haven, Connecticut, USA. Department of Molecular Microbiology and Immunology, Brown University, Providence, \\ Rhode Island, USA. ${ }^{3}$ Departments of Pathology, and Genetics, and ${ }^{4}$ Department of Thoracic Surgery, Yale School of Medicine, New Haven, Connecticut, USA. ${ }^{5}$ Department of Microbiology \\ and Immunology, University of North Carolina, Chapel Hill, North Carolina, USA. ${ }^{6}$ Pulmonary, Allergy and Critical Care Medicine, University of Pittsburgh Medical Center, Pittsburgh, Pennsylvania, USA. \\ ${ }^{7}$ Department of Internal Medicine and Environmental Health Center, Kangwon National University Hospital, School of Medicine, Kangwon National University, Chuncheon, South Korea. \\ ${ }^{8}$ Department of Pulmonary and Critical Care Medicine, and Clinical Research Center for Chronic Obstructive Airway Diseases, Asan Medical Center, University of Ulsan College of Medicine, Seoul, South Korea.
}

\begin{abstract}
Cigarette smoke (CS) and viruses promote the inflammation and remodeling associated with chronic obstructive pulmonary disease (COPD). The MAVS/RIG-I-like helicase (MAVS/RLH) pathway and inflammasome-dependent innate immune pathways are important mediators of these responses. At baseline, the MAVS/RLH pathway is suppressed, and this inhibition must be reversed to engender tissue effects; however, the mechanisms that mediate activation and repression of the pathway have not been defined. In addition, the regulation and contribution of MAVS/RLH signaling in CS-induced inflammation and remodeling responses and in the development of human COPD remain unaddressed. Here, we demonstrate that expression of NLRX1, which inhibits the MAVS/RLH pathway and regulates other innate immune responses, was markedly decreased in 3 independent cohorts of COPD patients. NLRX1 suppression correlated directly with disease severity and inversely with pulmonary function, quality of life, and prognosis. In murine models, CS inhibited NLRX1, and CS-induced inflammation, alveolar destruction, protease induction, structural cell apoptosis, and inflammasome activation were augmented in NLRX1-deficient animals. Conversely, MAVS deficiency abrogated this CS-induced inflammation and remodeling. Restoration of NLRX1 in CS-exposed animals ameliorated alveolar destruction. These data support a model in which CS-dependent NLRX1 inhibition facilitates MAVS/RHL activation and subsequent inflammation, remodeling, protease, cell death, and inflammasome responses.
\end{abstract}

\section{Introduction}

Chronic obstructive pulmonary disease (COPD) encompasses several clinical syndromes, most notably emphysema and chronic bronchitis. It represents a major unmet medical need and is strongly associated with cigarette smoke (CS) exposure. We previously demonstrated that CS and viruses utilize the RIG-I-like helicase $(\mathrm{RLH})$ innate immune pathway to generate COPD-like alveolar destruction and airway remodeling and that the mitochondrial antiviral signaling molecule (MAVS) plays a key role in these responses (1). Here, we hypothesized that the tissue effects of CS are mediated by its ability to abrogate the effects of specific MAVS inhibitors and that the nucleotide-binding domain and leucine-rich repeat-containing protein $\mathrm{X} 1$ (NLRX1) is a critical target of this CS-induced

Note regarding evaluation of this manuscript: Manuscripts authored by scientists associated with Duke University, The University of North Carolina at Chapel Hill, Duke-NUS, and the Sanford-Burnham Medical Research Institute are handled not by members of the editorial board but rather by the science editors, who consult with selected external editors and reviewers.

Conflict of interest: The authors have declared that no confict of interest exists. Submitted: January 20, 2015; Accepted: March 31, 2015

Reference information: / Clin Invest. 2015;125(6):2458-2462. doi:10.1172/JCI71747. disinhibition. To test this hypothesis, we evaluated the expression of NLRX1 in 3 cohorts of patients with COPD and characterized the relations between NLRX1 suppression and disease severity. In addition, we evaluated the effects of CS on the expression of NLRX1 and defined the roles of MAVS and NLRX1 in CS-induced murine pulmonary inflammation and alveolar remodeling responses.

\section{Results and Discussion}

We extracted the mRNAs from fresh-frozen lung tissues from the 7 controls and 36 patients with COPD in the NIH-sponsored Lung Tissue Research Consortium (LTRC) cohort. The latter contained 6, 10, 10, and 10 specimens from Global Initiative for Chronic Obstructive Lung Disease (GOLD) stage 1, 2, 3, and 4 individuals, respectively (Supplemental Table 1A; supplemental material available online with this article; doi:10.1172/JCI71747DS1). Real-time RT-PCR analysis demonstrated that the levels of mRNA encoding NLRX1 were significantly decreased in patients with advanced stages of COPD (Figure 1, A and B). Western blotevaluations demonstrated that NLRX1 protein expression was significantly decreased in patients with advanced stages of COPD (Supplemental Figure 1). $N L R X 1$ expression correlated significantly with the patient's forced expiratory volume in 1 second $\left(\mathrm{FEV}_{1} ; \%\right.$ predicted $)$, an indicator of 


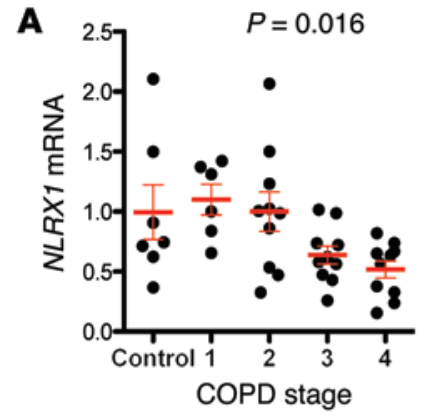

E

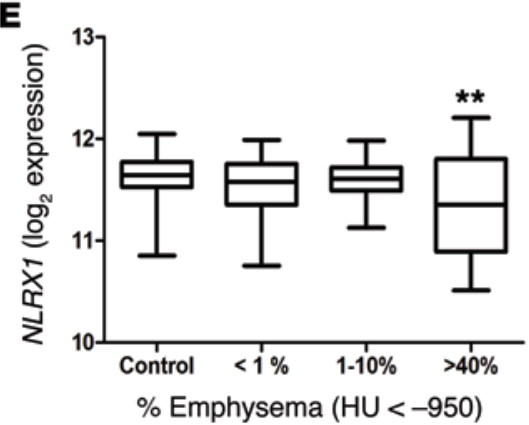

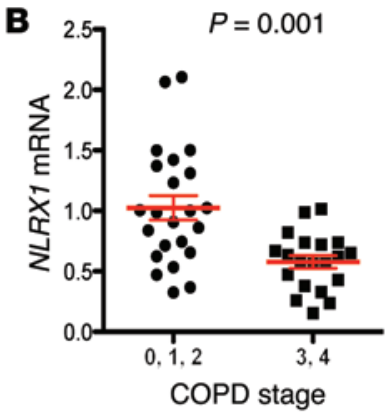

COPD stage
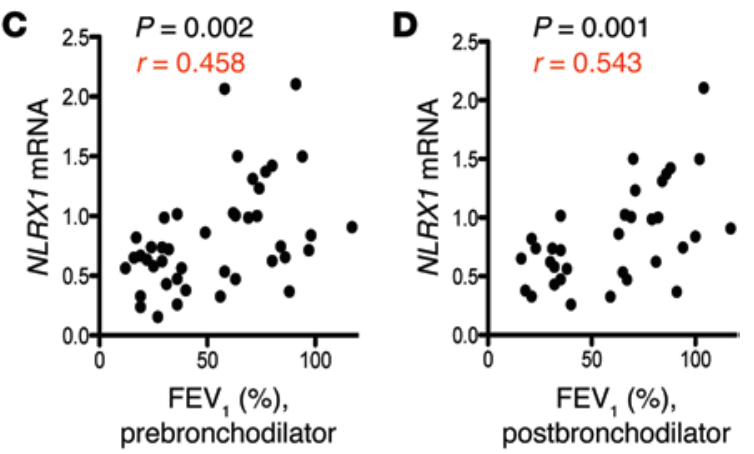

G

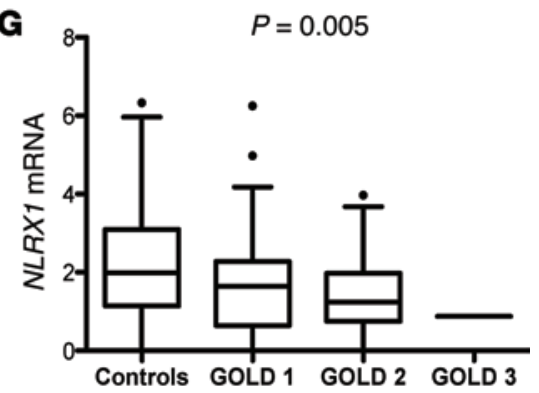

Figure 1. NLRX1 mRNA suppression in patients with COPD and its correlation with disease severity. (A) NLRX1 mRNA levels in LTRC samples were plotted for controls (0, no disease) and for patients with COPD of varying severity (COLD 1, 2, 3, and 4). (B) NLRX1 mRNA levels were compared in subjects with no disease (controls, 0 ) and in patients with mild-to-moderate (GOLD 1 and 2) and severe COPD (GOLD 3 and 4). (C and D) Correlation between NLRX1

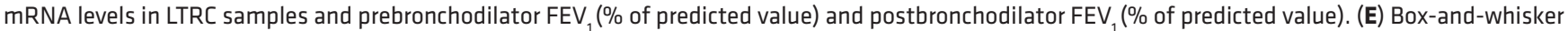
plot for the Pittsburgh cohort depicting the relation between NLRX1 gene expression and radiologic emphysema $\left({ }^{* *} P<0.01\right.$ compared with control and the $>40 \%$ emphysema group). (F and $\mathbf{G})$ From the Asan cohort, NLRX1 gene transcriptome levels were plotted for controls versus patients with COPD and for controls versus patients with COPD of varying severity (GOLD 1, 2, and 3). Red bars in A, B, and $\mathbf{G}$ represent the mean \pm SEM of the noted evaluations. Data were calculated using nonparametric Kruskal-Wallis (A), Mann-Whitney $U(\mathbf{B})$, Pearson's correlation (C and D), ANOVA (E and $\mathbf{C})$, and 2-tailed $t(\mathbf{F})$ tests. HU, Hounsfield units.

airflow limitation and a measure of COPD disease severity (Figure 1, C and D). Similar correlations were noted between NLRX1 suppression and pre- and postbronchodilator $\mathrm{FEV}_{1}$, suggesting that these relationships are not related to reversible airway obstruction (Figure 1, C and D). In addition, similar suppression of $N L R X 1$ was seen in a cohort of patients from the University of Pittsburgh (Supplemental Table 1B) and was most impressive in those with severe emphysema (Figure 1E). Because the LTRC and Pittsburgh cohorts did not contain large numbers of patients with mild-to-moderate disease, we also studied a third cohort of Korean patients with mild-to-moderate disease (Asan cohort) (Supplemental Table 1C). Evaluation of this Asan cohort demonstrated that NLRX1 expression was also significantly decreased in patients with GOLD stage 1 or 2 COPD, in whom it correlated significantly with the patient's $\mathrm{FEV}_{1}$ (\%, predicted) (Figure 1, F and G, Supplemental Figure 2, and Supplemental Figure 3, A and B). Almost all samples from the 3 human cohorts were from former or current smokers (Supplemental Table 1), preventing the evaluation of smoking effects on NLRX1 expression in these cohorts. There was no statistical difference in NLRX1 expression levels between former smokers and current smokers among the 3 human cohorts (Supplemental Figure 4 and data not shown). Finally, these alterations were at least partially NLRX1 specific, because the expression of related genes including RIGI, MDA5, MAVS, NLRP3, and caspases 1, 4, and 5 were not similarly altered (Supplemental Figure 5 and data not shown).
These studies demonstrate that NLRX1 expression is selectively decreased in patients with COPD where this suppression correlates with clinical stage and parameters of disease severity.

We next evaluated the relationships between NLRX1 gene expression and clinical parameters of COPD. In the LTRC cohort, NLRX1 gene expression levels correlated with other measures of pulmonary function including diffusing capacity $\left(\mathrm{DL}_{\mathrm{CO}}\right)$ and 6-minute walking distance (Supplemental Figure 6A and data not shown). The levels of NLRX1 mRNA correlated inversely with the BODE (BMI, airflow obstruction, dyspnea, and exercise) index and scores on the St. George's Respiratory Questionnaire (SGRQ) (Supplemental Figure 6B and data not shown), which are predictors of disease mortality and quality of life, respectively. NLRX1 also correlated inversely with dyspnea as measured with the Borg scale at the termination of exercise (Supplemental Figure 6C). CXCL13, which is produced by lymphoid follicles in COPD (2) and inhibited via an NLRX1-dependent mechanism (see below), was significantly enhanced in patients with advanced stages of COPD, where it correlated inversely with the expression of NLRX1 and the patient's $\mathrm{FEV}_{1}$ (\%, predicted), $\mathrm{DL}_{\mathrm{CO}}$, BODE index, and SGRQ scores (Supplemental Figures 7 and 8, and data not shown). These studies reinforce the relations between the suppression of NLRX1regulated pathways and abnormal pulmonary function and highlight the relations between NLRX1 suppression and mortality, poor quality of life, and exercise-induced dyspnea. 


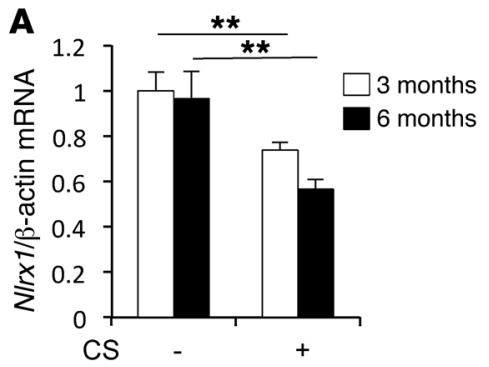

B

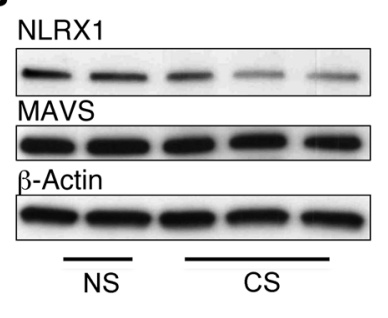

C

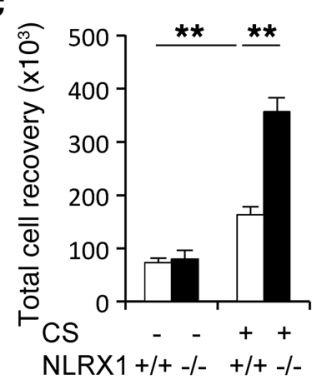

D

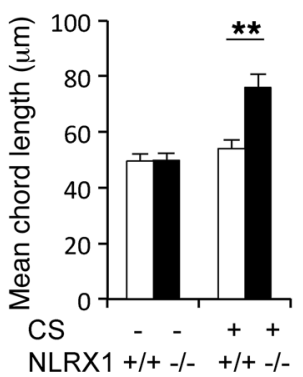

E

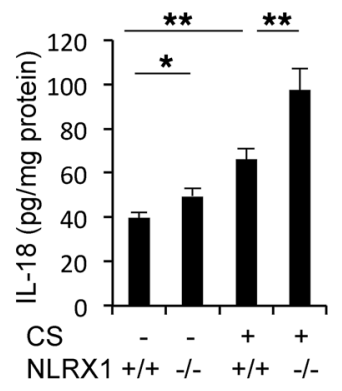

$\mathbf{F}$

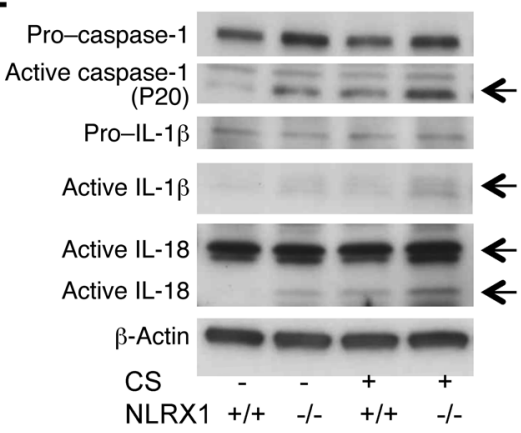

G

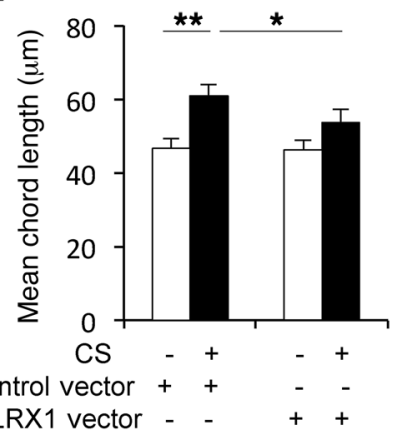

Figure 2. CS-induced suppression of NLRX1 and the role of NLRX1 in CS-induced pulmonary inflammation and alveolar destruction. (A) N/rx1 mRNA expression following CS exposure. After 3 months of CS exposure, (B) Western blot evaluation of NLRX1 in BAL cells, (C) BAL total cell recovery, (D) lung morphometry, (E) levels of active IL-18 in lung lysates measured by ELISA, and (F) activation status of IL-1 1 , IL-18, and caspase 1 in lung lysates. (G) Comparisons of the mean chord length in lungs from mice treated with lentiviral NLRX1 (NLRX1 vector ${ }^{+}$) and from controls (control vector ${ }^{+}$) after a 6-month exposure to CS. Data in $\mathbf{A}, \mathbf{C}, \mathbf{D}, \mathbf{E}$, and $\mathbf{G}$ represent the mean \pm SEM of evaluations in a minimum of 5 mice $\left({ }^{*} P<0.05\right.$; $\left.{ }^{*} P<0.01\right)$. B and $\mathbf{F}$ are representative of a minimum of 3 similar experiments. The 2-tailed $t$ test (A) and 2-way ANOVA (C-E and $\mathbf{G}$ ) were used.

To define the role(s) of NLRX1 in CS-induced responses, we characterized the effects of CS on NLRX1 expression in WT mice and defined the CS-induced tissue responses in WT and Nlrx1-null $\left(\mathrm{N} r l \times 1^{-/}\right)$mutant animals. Indeed, the levels of pulmonary Nlrx 1 mRNA and NLRX1 protein were significantly decreased after CS exposure (Figure 2A and Supplemental Figure 9). Immunohistochemical staining of NLRX1 protein revealed that NLRX1 expression was prominent in alveolar macrophages in both mice and humans (Supplemental Figures 10 and 11, respectively). Western blot analysis of NLRX1 protein in BAL cells from lungs exposed to CS for 3 months, where more than $95 \%$ of total BAL cells are alveolar macrophages, further confirmed that NLRX1 protein was significantly suppressed after CS exposure (Figure 2B and Supplemental Figure 12A). The expression of MAVS protein, however, was not significantly altered in BAL cells after CS exposure (Figure 2B and Supplemental Figure 12B). This suppression of NLRX1 was at least partially reversible, because Nlrx1 gene expression returned to normal levels 3 months after the cessation of CS exposure (Supplemental Figure 13). In keeping with reported experiments using WT mice, modest BAL and alveolar inflammation and modest alveolar destruction could be appreciated after 3 and 6 months of CS exposure, respectively (3). Importantly, these inflammatory and remodeling responses were markedly enhanced in lungs from CS-exposed $\mathrm{Nlrx}^{-/-}$mice, which manifested enhanced BAL total cell, macrophage, and neutrophil recovery (Figure $2 \mathrm{C}$ and data not shown) and exaggerated emphysema that could be seen with histologic and morphometric assessments after only 3 months of CS exposure (Figure 2D and Supplemental Figure 14).
To further define the mechanisms by which NLRX1 suppression mediates CS-induced pulmonary inflammation and alveolar remodeling responses, we evaluated the molecules and pathways that are believed to play important roles in COPD pathogenesis. In accord with the importance of protease-antiprotease balance, cell death, viral infection, and inflammasome activation in COPD pathogenesis (reviewed in refs. 4, 5), CS induction of Mmp12 (Supplemental Figure 15A), cathepsins K and S (data not shown), type 1 Ifns (Supplemental Figure 15B), epithelial DNA injury and cell death (Supplemental Figure 15C), and caspase 1, IL-1 $\beta$, and IL-18 activation (Figure 2, E and F, and Supplemental Figure 15D) was enhanced in Nlrx1-null mice compared with that observed in WT mice. Interestingly, CS exposure caused a modest increase in Cxcl13 in the lungs of WT mice, and this gene expression was synergistically upregulated in the lungs of NLRX1-deficient mice after 3 months of CS exposure (Supplemental Figure 16).

Finally, we performed studies to determine whether NLRX1 supplementation could abrogate the phenotype in our CS-exposed mice in vivo. In these experiments, we used a lentiviral gene delivery system and exposed mice that had been given NLRX1 or controls to CS for 6 months. These experiments demonstrated that CS-induced emphysematous alveolar destruction was markedly decreased in lungs of mice with NLRX1 overexpression compared with that seen in control lungs (Figure $2 \mathrm{G}$ and Supplemental Figure 17). Taken together, these results suggest that NLRX1 is a critical inhibitor of CS-induced pulmonary inflammation and tissue remodeling responses and that its inhibitory functions are decreased in pulmonary tissues by chronic CS exposure. 
A

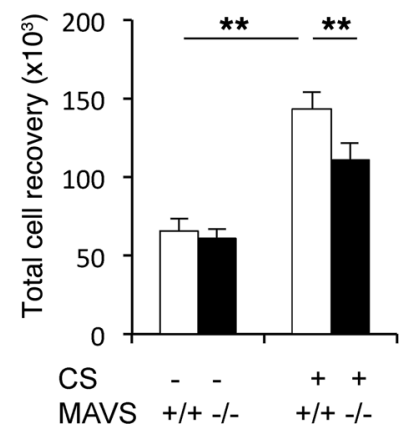

D

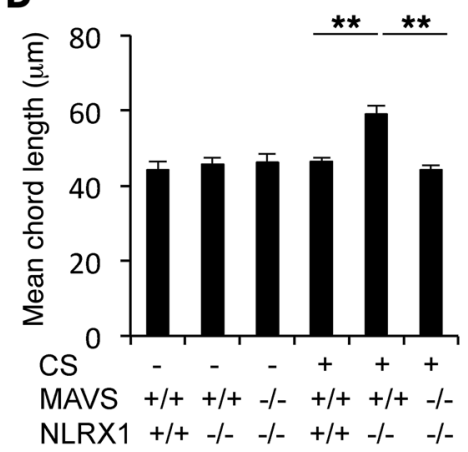

B

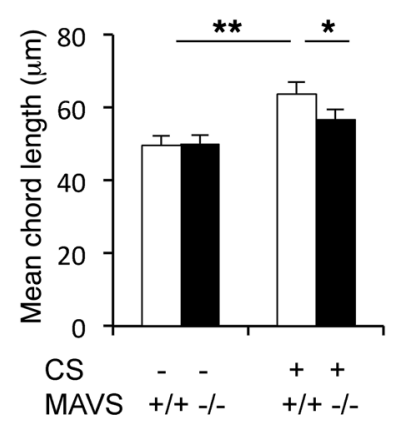

E

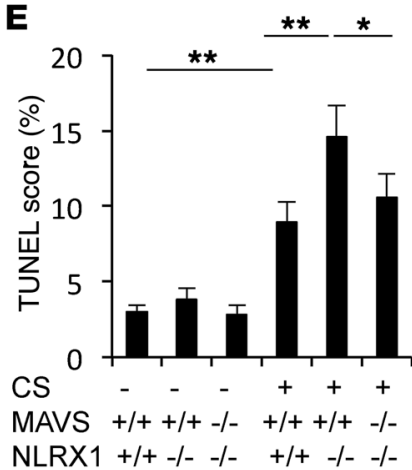

C

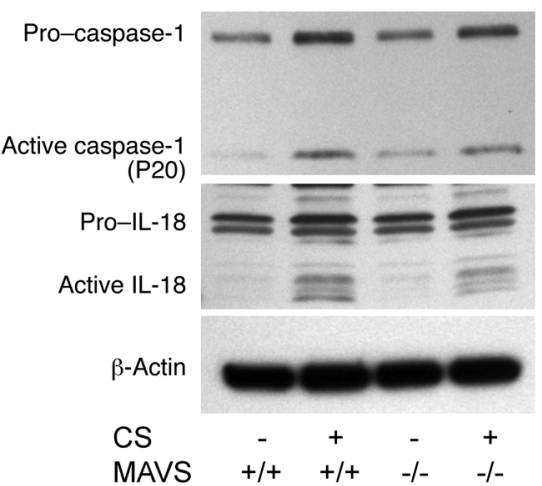

$\mathbf{F}$

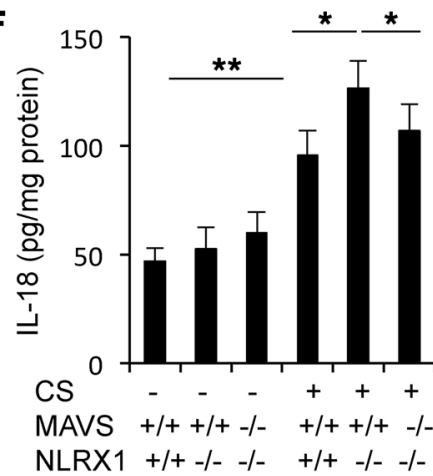

Figure 3. Role of MAVS in CS-induced pulmonary inflammation and alveolar destruction. After 6 months of CS exposure, the role of MAVS was evaluated. (A) BAL total cell recovery and (B) mean chord length. (C) Western blot analyses of lung lysates were used to demonstrate the activation status of IL-18 and caspase 1. (D-F) Results of the evaluation of mean chord length, TUNEL staining of structural cells, and IL-18 levels in lung tissues from Mavs ${ }^{-/-} \mathrm{Nlrx}^{-/-}$, NIrx $7^{-1-}$, and WT control $\left(\mathrm{Mavs}^{+/+} \mathrm{Nlrx}^{1^{+/+}}\right)$mice after a 3-month exposure to CS. Values in A, B, D, and $\mathbf{F}$ represent the mean \pm SEM of evaluations in a minimum of 5 mice $\left({ }^{*} P<0.05 ;{ }^{* *} P<0.01\right)$. Panel $\mathbf{C}$ is representative of a minimum of 3 similar evaluations. A 2-way ANOVA test was used for $\mathbf{A}, \mathbf{B}, \mathbf{D}$, and $\mathbf{F}$.

The studies noted above demonstrate that CS exposure decreases NLRX1 expression, and NLRX1 has been reported to be an inhibitor of MAVS $(6,7)$. Thus, we next conducted studies to determine whether mitochondria, in particular MAVS, play an important effector role in CS-induced inflammation and remodeling. First, we compared CS-induced responses in WT mice with those in $\mathrm{Mavs}^{-/}$mice. We found that inflammation and alveolar destruction were significantly decreased in lungs from CS-exposed $\mathrm{Mavs}^{-/}$mice compared with WT controls (Figure 3, A and B, and Supplemental Figure 18). CS-induced activation of caspase 1 , protease responses, IL-1 $\beta$ and IL-18, TUNEL staining, and type 1 IFN production were also diminished in the absence of MAVS (Figure 3C, Supplemental Figure 19, and data not shown). In contrast, CS-induced suppression of NLRX1 was not altered in the lungs of $\mathrm{Mavs}^{-1-}$ mice exposed to CS for up to 6 months (Supplemental Figure 19C). Next, to determine whether MAVS played a critical role in the enhancement of CS-induced inflammation and alveolar destruction observed in $\mathrm{Nlrx}^{-/-}$mice, we exposed NLRX1 and MAVS double-mutant ( $\left.\mathrm{NrXx}^{-1-} \mathrm{Mavs}^{--}\right)$ mice and appropriate controls to CS for 3 months. These studies demonstrated that the enhanced emphysema, cell death, IL-18 production, and CXCL13 production observed in NLRX1 single-null mice exposed to CS were decreased in similarly exposed double-mutant mice (Figure 3, D-F, and Supplemental Figures 20 and 21). Overall, these studies demonstrate that MAVS plays a critical role in the exaggerated inflammatory, remodeling, proteolytic, cell death, and cytokine responses that are induced by CS in states of NLRX1 deficiency.

Studies of COPD and models of this disorder have highlighted the importance of antiviral innate immunity, protease excess, epithelial cell death, and inflammasome activation in disease pathogenesis $(1,3-5)$. Our studies add to and integrate these findings by demonstrating that mitochondria play an important role in the regulation and effector phases of CS-induced responses. Specifically, they demonstrate that NLRX1 is an important inhibitor of MAVS at baseline and that CS stimulates inflammation and remodeling by suppressing NLRX1. Inhibition of NLRX1 augmented antiviral innate immune responses, protease expression, cell death, and inflammasome activation, thereby mechanistically integrating the present and previous observations. The disease relevance of these studies was also confirmed in studies demonstrating that NLRX1 expression is decreased in the lungs of patients with COPD, where this suppression correlates with disease severity, abnormal pulmonary function, decreased quality of life, and poor prognosis.

NLRX1 is a member of the Nod-like receptor family of intracellular sensors of microbial- and danger-associated molecular patterns $(7,8)$. In early studies, NLRX1 was identified to be a negative regulator of MAVS, a key adapter molecule of MAVS/RLH antiviral signaling (7). It is now known to be a negative modulator of LPS-induced TRAF6/NK-кB signaling and has been shown to 
regulate autophagy $(6,9,10)$, bind to UQCRC2 in mitochondrial respiratory chain complex III, and regulate mitochondrial production of ROS (11). This effector repertoire might have significant implications for our studies, because defects in autophagy/ mitophagy lead to the accumulation of damaged, ROS-producing mitochondria, which induce inflammasome activation (12). In accord with this concept, a recent report demonstrated that MAVS also plays a role as a critical adaptor molecule in inflammasome activation (13). Taken together, these studies suggest that NLRX1 inhibition of MAVS-dependent inflammasome activation plays an important role in the pathogenesis of COPD. Additional investigation will be required to define the mechanism(s) that CS uses to inhibit NLRX1, the mechanism(s) that NLRX1 uses to control CSand MAVS-dependent responses, and the pathway(s) that dominate in the generation of COPD.

In summary, these studies demonstrate that the suppression of NLRX1 is a critical event in CS-induced responses. They also raise the possibility that the degree of NLRX1 suppression may be an important index of COPD severity and that polymorphisms or other alterations that decrease NLRX1 function can contribute to disease susceptibility. Importantly, the demonstration that NLRX1 supplementation abrogates CS-induced alveolar destruction also suggests that interventions that prevent the inhibition of NLRX1, augment the effects of NLRX1, abrogate MAVS function, or restore NLRX1 suppression may be therapeutically useful in this disease. Additional investigation of these possibilities in COPD and other CS-related diseases is warranted.

\section{Methods}

For more detailed information, see the Supplemental Methods.

Study populations. Three human cohorts from North America and Asia were evaluated in these studies.

Statistics. All data represent the mean \pm SEM. Statistical significance was defined at a $P$ value of 0.05 or less. All analyses were performed using SPSS software, version 22.0 (IBM Corporation).

Study approval. All human studies were approved by the Human Investigation Committee (HIC) of Yale University (HIC 110508577) and the LTRC, a nationwide resource program of the National Heart, Lung, and Blood Institute (NHLBI) (LTRC 11-99-0005). All in vivo experiments in animals were approved by the Yale Animal Care and Use Committee (YACUC; 2011-07586).

\section{Acknowledgments}

This work was supported by Flight Attendants Medical Research Institute (FAMRI) grants 13258 (to M.J. Kang) and 82571 (to J.A. Elias); Connecticut Department of Public Health grant 2013-0196 (to M.J. Kang and J.A. Elias); NIH grants R56HL119511 (to M.J. Kang), HL079328 and PO1 HL114501 (to J.A. Elias), AG047632 (to G.S. Shadel), and U19-AI1077437 (to J.P. Ting); and American Cancer Society grant PF-13-035-01-DMC (to A.P. West).

Address correspondence to: Jack A. Elias, Division of Medicine and Biological Sciences, Brown University, Warren Alpert School of Medicine, Box G-A1, 97 Waterman Street, Providence, Rhode Island 02912, USA. Phone: 401.863.3336; E-mail: jack_elias@brown.edu.
1. Kang MJ, et al. Cigarette smoke selectively enhances viral PAMP- and virus-induced pulmonary innate immune and remodeling responses in mice. J Clin Invest. 2008;118(8):2771-2784.

2. Litsiou E, et al. CXCL13 production in B cells via Toll-like receptor/lymphotoxin receptor signaling is involved in lymphoid neogenesis in chronic obstructive pulmonary disease. Am J Respir Crit Care Med. 2013;187(11):1194-1202.

3. Kang MJ, et al. IL-18 is induced and IL-18 receptor alpha plays a critical role in the pathogenesis of cigarette smoke-induced pulmonary emphysema and inflammation. JImmunol. 2007;178(3):1948-1959.

4. Tuder RM, Petrache I. Pathogenesis of chronic obstructive pulmonary disease. J Clin Invest. 2012;122(8):2749-2755.
5. Decramer M, Janssens W, Miravitlles M. Chronic obstructive pulmonary disease. Lancet. 2012;379(9823):1341-1351.

6. Allen IC, et al. NLRX1 protein attenuates inflammatory responses to infection by interfering with the RIG-I-MAVS and TRAF6-NF- $\kappa$ B signaling pathways. Immunity. 2011;34(6):854-865.

7. Moore CB, et al. NLRX1 is a regulator of mitochondrial antiviral immunity. Nature. 2008;451(7178):573-577.

8. Davis BK, Wen H, Ting JP. The inflammasome NLRs in immunity, inflammation, and associated diseases. Annu Rev Immunol. 2011;29:707-735.

9. Xia X, et al. NLRX1 negatively regulates TLR-induced NF-kappaB signaling by targeting TRAF6 and IKK. Immunity. 2011;34(6):843-853.
10. Lei Y, et al. The mitochondrial proteins NLRX1 and TUFM form a complex that regulates type I interferon and autophagy. Immunity. 2012;36(6):933-946.

11. Arnoult D, Soares F, Tattoli I, Castanier C, Philpott DJ, Girardin SE. An N-terminal addressing sequence targets NLRX1 to the mitochondrial matrix. J Cell Sci. 2009;122(pt 17):3161-3168.

12. Zhou R, Yazdi AS, Menu P, Tschopp J. A role for mitochondria in NLRP3 inflammasome activation. Nature. 2011;469(7329):221-225.

13. Subramanian $\mathrm{N}$, Natarajan K, Clatworthy MR, Wang Z, Germain RN. The adaptor MAVS promotes NLRP3 mitochondrial localization and inflammasome activation. Cell. 2013;153(2):348-361. 